\title{
LA FORMACIÓN DE LOS EDUCADORES EN ALBERGUES INFANTILES EN MÉXICO. FACTOR DE RIESGO PARA LA CONDUCTA ANTISOCIAL DE LOS NIÑOS
}

TRAINING OF EDUCATORS IN CHILDREN'S SHELTERS IN MÉXICO. RISK FACTOR FOR ANTISOCIAL BEHAVIOR IN CHILDREN A FORMAÇÃO DE PROFESSORES EM ABRIGOS INFANTIS NO MÉXICO. FATOR DE RISCO PARA COMPORTAMENTO ANTI-SOCIAL DE CRIANÇAS DAS FAMÍLIAS.

PALABRAS CLAVES: Protección infantil acogimiento residencial maltrato infantil conducta antisocial educadores
Fecha de recepción del artículo: 27.III.2015

Fecha de revisión del artículo: 1.IV.2015 Fecha de aceptación final: 25.1.2016

\begin{tabular}{|l|l|}
\hline $\begin{array}{l}\text { PALABRAS CLAVES: } \\
\text { Protección infantil } \\
\text { acogimiento }\end{array}$ & $\begin{array}{l}\text { RESUMEN: En México, los albergues infantiles son requeridos para garantizar niveles míni- } \\
\text { mos de bienestar a niños(as) víctimas. En estos albergues, los educadores adquieren mucha } \\
\text { importancia. Por esto, el objetivo general del estudio fue analizar la formación de los educa- } \\
\text { maltrato infantil } \\
\text { conducta antisocial } \\
\text { educadores albergues infantiles y su relación con el ambiente y comportamiento antisocial de } \\
\text { los menores. Se aplicó la Escalas ELES a i17 educadores de albergues infantiles al Norte de }\end{array}$ \\
$\begin{array}{l}\text { México y la escala Conners para Profesores a los maestros de } 233 \text { menores albergados. El } \\
\text { modelo probado mostró que el factor Ambiente influye de manera directa y negativa sobre } \\
\text { el de Conducta Antisocial del menor y el factor de Competencias de Educadores produce } \\
\text { un efecto directo y positivo sobre el Ambiente. Sin embargo, las competencias del educador } \\
\text { no afectaron directamente la conducta de los(as) niños(as), como se esperaba, sino que lo } \\
\text { afectan de manera indirecta a través del ambiente. }\end{array}$ \\
\hline
\end{tabular}




\begin{tabular}{|c|c|}
\hline $\begin{array}{l}\text { KEY WORDS: } \\
\text { Child welfare } \\
\text { foster care } \\
\text { child abuse } \\
\text { antisocial conduct } \\
\text { educators }\end{array}$ & $\begin{array}{l}\text { ABSTRACT: In Mexico, children 's shelters are required as a measure to guarantee minimum } \\
\text { levels of well-being for abused or neglected children. At these shelters, the presence of edu- } \\
\text { cators is fundamental. Therefore, the objective of this study was to analyze the training edu- } \\
\text { cators working at shelters for children and its relationship with the shelter's environment and } \\
\text { the antisocial behavior of children. ELES was administered to } 117 \text { educators at the shelter in } \\
\text { the North part of Mexico and Conners for Teachers Scale to the school teachers of } 233 \text { chil- } \\
\text { dren living at those shelters at that time. According to the results from the tested model, the } \\
\text { Environment factor had a direct and negative influence on antisocial Behavior, and the Educa- } \\
\text { tor's Competences factor had a direct and positive influence on the Environment. However, } \\
\text { the educators' competences had no significant effect on the children's antisocial behavior, but } \\
\text { affected it indirectly through the environment. }\end{array}$ \\
\hline $\begin{array}{l}\text { PALAVRAS-CHAVE: } \\
\text { Proteção à criança } \\
\text { Colocação } \\
\text { residencial } \\
\text { criança maltratada } \\
\text { comportamento } \\
\text { anti-social } \\
\text { educadores }\end{array}$ & $\begin{array}{l}\text { RESUMO: No México, os abrigos infantis são necessários para garantir níveis mínimos de } \\
\text { bem-estar às crianças (vítimas). Estes abrigos educadores adquirem muita importância. Por- } \\
\text { tanto, o objetivo geral do estudo foi analisar a formação de educadores em abrigos de crian- } \\
\text { ças e sua relação com o meio ambiente e comportamento anti-social de menores. A escala } \\
\text { ELES foi aplicada a } 117 \text { educadores de abrigos de crianças no norte do México e a escala } \\
\text { de Conners foi administrada a professores de } 233 \text { crianças alojados em abrigos infantis. O } \\
\text { modelo testado mostrou que a influência de fator de ambiente es direta e negativa sobre o } \\
\text { comportamento anti-social dos menores e a competência de fator de educadores produz um } \\
\text { efeito direto e positivo sobre o meio ambiente. No entanto, as competências do educador } \\
\text { não afetou diretamente a conduta das crianças, conforme o esperado, mas afectam indirec- } \\
\text { tamente através da ambiente }\end{array}$ \\
\hline
\end{tabular}

\section{Aproximación teórica}

Existen en el mundo 100 millones de niñas, niños y adolescentes que trabajan y viven en la calle. De éstos, aproximadamente 40 millones se encuentran en Latinoamérica, ocupando México el segundo lugar (Hernández, 2014). No obstante, de acuerdo a varias fuentes (Gaxiola \& Frías, 2008; González, 2010; INMUJERES, 2006; UNICEF, 2009a, 2009b) la ausencia de estadísticas confiables es un problema en lo que se refiere a los menores de edad en situación de riesgo.

Al hablar específicamente de menores maltratados, las cifras también varían considerablemente ya que no hay datos fidedignos que muestren su magnitud, en particular porque la mayoría de las violaciones a los derechos de los niños son difíciles de medir (UNICEF, 2009b). El número de denuncias recibidas en México por maltrato infantil entre 1995 y 2007, pasó de 15,391 a 43,985 y tan sólo del 2006 al 2008, se reportaron a nivel nacional, 23 mil homicidios de infantes y adolescentes de ambos sexos (INEGI, 2010a). Otro estudio, realizado en el 2002 por el Sistema de Desarrollo Integral de la Familia (DIF) y el Programa de Prevención al Maltrato Infantil, menciona haber recibido en ese año 23,585 denuncias por maltrato infantil de las cuales únicamente 13,332 fueron comprobadas (Gaxiola \& Frías, 2008).

Las leyes que forman el marco jurídico mexicano sobre el que deben trabajar estas instituciones van desde la Ley Orgánica de la Procuraduría de la Defensa del Menor y la Familia (1971), hasta la Norma Oficial Mexicana para la Prestación de Servicios de Asistencia Social para Menores y
Adultos Mayores (1997). Aunque las normas nacionales aplican tanto para las instituciones privadas como para las instituciones gubernamentales (Ley de Asistencia Social del Estado de Sonora, 1986) no se establecen lineamientos en cuanto al uso de programas de readaptación, desempeño educativo por parte del personal, ni sobre el proceso de selección del mismo. Esto, aunado a la falta de datos confiables y las investigaciones a los albergues infantiles llevadas a cabo en distintos lugares de la República, ha ocasionado que algunos Estados busquen modificar su propia legislación.

En México se han encontrado en funcionamiento albergues insalubres, con personal no capacitado, con una alta proporción de niños por educador, y carentes de todo tipo de programas de trabajo (CEDHJ, 2006). Algunos autores consideran que los menores en las instituciones Ilegan a sufrir maltrato físico, psicológico y sexual. Además, se considera maltrato, la falta de protección adecuada o la atención necesaria, ya sea por la misma dinámica del albergue o por negligencia (Redondo, Muñoz \& Torres Gómez, 1998). A esta falta de cobertura de las necesidades elementales, aunado al hacinamiento y la indefensión "ante medidas y procedimientos arbitrarios o maltratantes por parte del personal en materia de sanciones o limitaciones injustificadas" (Fuertes \& Fernández del Valle, 2001, p. 48) se le conoce como maltrato institucional.

\section{La figura del Educador}

Una de las privaciones más serias experimentadas durante la institucionalización es la falta de un cuidador consistente y sensible con quien el niño 
pueda formar un apego saludable y confiable (Gribble, 2007) que le asegure lazos afectivos de calidad (Barudy, 2005b). A esta persona también se le conoce como adulto significativo (Barudy, 2005a, Melendro, 2007), adulto alternativo (Arruabarrena, 2001) o persona de referencia (Bravo \& Fernández del Valle, 2009). Sin embargo, esto no siempre es tomado en cuenta por las administraciones o el sistema judicial (Barudy, 2005b).

En una valoración realizada por ex residentes de los centros (García-Barriocanal, De la Herrán \& Imaña, 2007) se estima como positivo el apoyo y buen trato recibido por parte de los educadores, la educación recibida, las semejanzas entre el albergue y un ambiente familiar y el ambiente estructurado con normas claras y espacios personalizados.

Para que los jóvenes se incorporen a una dinámica de proximidad y afecto que les ayude a establecer esta relación duradera con sus educadores es necesario mantener un clima de respeto (Melendro, 2007), cálido y acogedor (Bravo \& Fernández del Valle, 2009; Nesmith, 2006) que facilite "situaciones que permitan y estimulen las relaciones interpersonales y contribuyan a que la infancia acogida construya una imagen fiel y positiva de sí misma" (Cruz, 2009, p. 589). El niño o adolescente ha de sentir el valor de la estabilidad y consistencia en los cuidados del educador (Papalia, Wendkos \& Duskin, 2001) y recibir reacciones sanas y espontáneas hacia su conducta, de tal manera que pueda realizar los ajustes personales y de comportamiento necesarios para alcanzar una convivencia gratificante que le permita generar, a partir de aquí, "esquemas relacionales nuevos y utilizables" (Mondragón \& Trigueros, 2004, p. 196) para el futuro.

Para algunos, lo que convierte al profesional en tal es la capacitación para realizar su tarea y la solvencia de su formación (Núñez, 1993). Cuando un educador carece de conocimiento y recursos técnicos "suele operar él mismo (aun sin saberlo), como barrera de contención. En ello suele poner el cuerpo, en lugar de un aparato conceptual adecuado" (Núñez, 1993, p. 136). De aquí que para Petrus (1997, p. 9) un número importante de fracasos en la intervención se deban a una "inadecuada capacitación personal para ubicarse frente a los problemas sociales". Se desprenden entonces, que una de las tendencias de futuro a nivel internacional sea la relativa a los cambios en materia de personal, a la profesión de los trabajadores que atienden directamente a los niños y a otorgar mayor importancia a la formación permanente de los mismos (Fernández del Valle \& Fuertes, 2007).

A este respecto, algunos países de Europa y de América Latina, han llevado a cabo esfuerzos por delimitar un profesional capaz y bien entrenado para atender de manera directa a los grupos vulnerables, incluidos los niños, adolescentes y adultos (AIEJI, 2007) desde una perspectiva de educación para la vida, denominado "educador social". Asociaciones como la Internacional de Educadores Sociales (AIEJI) fundada en Alemania hace más de medio siglo y la Asociación Estatal de Educación Social (ASEDES) en Barcelona, entre otras, han trabajado arduamente en la creación de un marco teórico, metodológico y jurídico para una educación concebida como "la teoría de cómo las condiciones psicológicas, sociales, materiales y diferentes orientaciones de valores promueven o dificultan el desarrollo, el crecimiento, la calidad de vida y el bienestar del individuo o del grupo" (AIEJI, 2005, p. 5), denominada educación social que, de acuerdo a Camors (2005) es una "perspectiva particular de la educación general” (p. 14).

\section{La Educación Social}

De manera general, la Educación Social se reflexiona como fenómeno, realidad y profesión mientras que la Pedagogía Social, como reflexión y disciplina científica (Cruz, 2009) "que considera, conceptúa e investiga esa educación social" (Ortega, 2005, p. 114).

La vivencia latinoamericana de la Pedagogía Social y la Educación Social no ha "podido delimitar y separar "lo formal" de lo "no formal" en su configuración práctica” (Vélez, 2006, p. 14). La influencia Centroeuropea y Germánica que han tenido estos países ha sido diversa según se observe desde cada uno de ellos. Se pudiera mencionar a Freire en Brasil con su educación para la liberación y la animación sociocultural de Ander-Egg en Argentina (Sáez, 2007). No obstante, y a pesar de que el trabajo en Educación Social lleva décadas de gestación, la profesionalización y reconocimiento profesional de los educadores sociales ha sido más reciente.

Poco a poco y de manera relativamente rápi$\mathrm{da}$, las recomendaciones internacionales y las acciones para adaptarlas han llegado a América. En México, sin embargo, los educadores de albergues infantiles no cuentan con un reconocimiento profesional (Núñez, 2008). Algunos no poseen títulos de educación superior y, en el mejor de los casos, provienen de perfiles académicos variados, agrupándolos por denominaciones que tienen que ver más con su posición dentro del organigrama institucional que con las funciones que desempeñan.

\section{Metodología}

El objetivo general de la presente investigación fue realizar un estudio sobre la formación de los educadores de los albergues infantiles y su 
relación con el ambiente y comportamiento de los menores. El primer objetivo específico fue identificar características generales sobre la formación de los educadores. El segundo objetivo fue determinar las características del ambiente dentro de cada albergue infantil con relación al lenguaje y la satisfacción del personal. El último, fue identificar los principales problemas conductuales de los menores albergados. Las hipótesis principales fueron: 1) una formación adecuada del educador, tanto en delimitación de sus funciones como en habilidades y actitudes, tendrá un efecto directo y positivo sobre el ambiente institucional, y 2) una formación adecuada del educador, tanto en delimitación de sus funciones como en habilidades y actitudes, tendrá efectos directos y negativos sobre los problemas conductuales del menor.

\subsection{Muestra}

El diseño de investigación es no-experimental, transversal, retrospectivo, con 10 muestras independientes que se tomaron de las 7 instituciones de asistencia social privada y 3 instituciones de asistencia social pública dedicadas al albergue de menores en situación de riesgo o pobreza de la ciudad de Hermosillo, Sonora, al norte de México. Los participantes estuvieron conformados por dos grupos: todos los educadores (117) de las instituciones que atienden a los menores de Hermosillo y la tercera parte de la población de menores de cada una de ellas. El personal de cada una de las instituciones varía según el presupuesto y el número de beneficiarios que tiene. En el estudio se consideraron a todos los educadores sociales que cada uno de los albergues tenía en el momento del levantamiento de datos. La media de edad de los educadores fue de 38 años, con una desviación estándar de 10.93. Sin embargo, en algunos albergues la media supera los 50 años de edad. La mayoría de los Educadores (77\%) son mujeres y sólo 27 del total, son varones.

La población de menores en todas las Instituciones contempladas en este estudio se concentró en el rango de primaria, si bien no fue exclusiva. Se eligieron también a menores de secundaria y bachiller, un total de 233 menores de ambos sexos, de 5 a los 17 años de edad. Con la finalidad de conocer sus comportamientos en el ambiente escolar, se aplicó a alguno de sus profesores de escuela la escala Conners.

\subsection{Instrumentos para la recogida de datos}

Para el estudio de la formación de los educadores se elaboró una escala de evaluación basada en los indicadores establecidos por el Libro Blanco de
Título de Grado en Pedagogía y Educación Social (ANECA, 2005) con modificaciones. Se escogieron algunas de las competencias de entre las 19 seleccionadas por los tres perfiles que convergen en la labor realizada con los menores en los albergues: a) Educador en procesos de intervención social; b) Educador de instituciones de atención e inserción social y c) Educador en procesos de acogida y adopción. La razón para la reducción de las competencias fue doble. La primera, el instrumento no podía ser muy extenso debido al tiempo que los educadores tenían disponible y segundo, esas competencias están basadas en la formación recibida en la Diplomaturas europeas. En México, debido a que no hay una licenciatura con sendas características y que los educadores actuales no han sido entrenados en ellas, carece de sentido tratar de evaluar competencias. Es por estos motivos que sólo se eligieron dos competencias transversales y una específica.

La elaboración del instrumento "Evaluación de la Labor del Educador Social” (ELES), se llevó a cabo en etapas. La primera de ellas fue un sondeo realizado en los diez albergues infantiles de Hermosillo. Por medio de visitas y llamadas telefónicas a cada institución se recopiló información general acerca del número de educadores, requisitos de contratación, perfiles y cursos de capacitación recibidos. Con base en esta información y en las competencias propuestas por ANECA, se elaboró el instrumento formado por cinco áreas o dimensiones:

Actitudes. La escala está formada por 4 ítems basados en el Cuestionario para Profesores (Doménech, Esbrí, González \& Miret, 2004) con una confiabilidad interna de .66. El cuestionario está diseñado para medir las actitudes de los profesores hacia los niños con necesidades educativas especiales derivadas de discapacidad, por lo que se realizaron algunos cambios en los reactivos.

Funciones. Se tomaron 7 de las funciones del Educador Social propuestas por Aneca (2005) y Vallés (2009) como fueron utilizados en el inventario utilizado por Escarbajal (2009) en su Modelo de Cuestionario para medir los objetivos de actuación profesional, que reportó un alfa de .86 .

Percepción. Se tomaron 8 ítems utilizados por Escarbajal (2009) en su Modelo de Cuestionario para medir los objetivos de actuación profesional con una confiabilidad de .93.

En un segundo momento la prueba se aplicó a 32 maestros de primaria y preescolar, de cuatro escuelas de Hermosillo. La prueba contenía las áreas anteriores y 13 ítems más comprendidos en las dimensiones:

Compromiso con la propia identidad. Consta de 4 ítems tomados de la Escala de Clima Organizacional con modificaciones, con alfa de .60. La 
escala utilizada es tipo Likert de 0 a 4 donde 0 equivale a nada y 4 siempre.

Habilidades. Se tomaron 9 ítems de la escala de Competencias de Comunicación Interpersonal (Rubin \& Martin, 1994) con alfa de .77. Los reactivos están diseñados para medir las habilidades de empatía, asertividad y expresividad con la misma escala Likert.

Ambiente. Se agregaron 5 ítems de la Early Chilhood Environment Rating Scale - Revised (ECERS-R) (Harms, Clifford \& Cryer, 2005) con alfa de entre .86 a .90 según la población a la que le sea aplicada. Se eligieron las dimensiones de Lenguaje y Razonamiento, de la que se excluyeron la mitad de los ítems, dando un total de 2, y 4 ítems de la dimensión Satisfacción de Necesidades, a la que se le realizaron pequeñas modificaciones con el fin de medir el grado de satisfacción que el educador tiene hacia su institución. Todos los reactivos fueron estructurados para ser medidos con escala de 0 a 6 , donde 0 equivale a Inadecuado y 6 a Excelente. La escala final quedó conformada como un instrumento de auto-reporte de 24 reactivos distribuidos en dos factores: el factor de competencias del educador, compuesto por las dimensiones de habilidades, funciones educativas, funciones administrativas, actitudes, percepción y compromiso con la propia identidad y el factor ambiente institucional conformado por las variables satisfacción de las necesidades del personal y lenguaje y razonamiento.

También se utilizó el Cuestionario de Conners para Profesores, en su versión abreviada (Conners, 2008), del que se utilizaron 9 ítems. Esta escala consta originalmente de 28 reactivos y ha mostrado una consistencia interna de .87. Cada pregunta describe una conducta característica que el profesor valoró según la intensidad con la que ésta se presenta en una escala de cuatro grados donde 0 equivale a nada, 1 a poco, 2 a bastante y 3 a mucho.

\subsection{Procedimiento}

En cada una de las instituciones se concertó una entrevista con el fin de solicitar permiso para realizar el estudio y explicar los objetivos del mismo. Los educadores dispusieron de un tiempo y lugar determinados para contestar, de manera individual, a las preguntas de la Escala, lo que les tomó alrededor de 25 minutos.

Para la evaluación de la conducta del niño, se eligió al azar a la tercera parte de la población de cada institución. La Escala de Conners para cada niño fue contestada por uno de sus profesores y tuvo una duración de 15 minutos. Un total de 20 escuelas fueron visitadas. A todos se les informó el objetivo de la investigación, y se les pidió su consentimiento informado, igualmente se les comunicó que podían dejar de contestar el cuestionario en cualquier momento si alguna pregunta les parecía inconveniente. Los instrumentos fueron aplicados por una estudiante de maestría en innovación educativa.

\subsection{Análisis de datos}

Por medio del paquete estadístico SPSS, se realizaron análisis univariados que incluían medias y desviaciones estándar para las variables continuas, frecuencias para las variables categóricas y alfas de Cronbach para determinar su confiabilidad (consistencia interna). Con el fin de agrupar las variables, se formaron índices con el promedio de cada uno de los reactivos de las escalas incluidas en el estudio.

Como paso final, se llevó a cabo un análisis de ecuaciones estructurales. Para determinar la pertinencia del modelo planteado se utilizaron indicadores de bondad de ajuste. En este estudio se utilizaron dos indicadores: el estadístico Chi cuadrada $\left(X^{2}\right)$ y los indicadores prácticos. La $X^{2}$ determina la diferencia entre el modelo propuesto y un modelo inclusivo. Si el modelo hipotético resulta pertinente, la $X^{2}$ tendrá un valor bajo y no significativo ( $p>05)$. Los indicadores prácticos se derivan de la $X^{2}$ y son el Índice Bentler-Bonnett de Ajuste No Normado (BBNNFI) y el Índice de Ajuste Comparativo (CFI) que tienen un mínimo aceptable de .90. Se incluyó también el indicador de la Raíz Cuadrada del Cuadrado Medio del Error de Aproximación (RMSEA) que deberá tener un valor menor a .08.

\section{Interpretación de los resultados}

Aun cuando $42 \%$ (de un total de 50 ) de los educadores tienen carreras técnicas o licenciaturas relacionadas con el trabajo de los albergues, sólo en 38 de ellos sus estudios están relacionados directamente con la atención a menores. El resto lo conforman licenciaturas como Derecho, Enfermería y Trabajo Social.

A pesar de que sólo 4 de los albergues son mixtos, éstos concentran la mayor población (67.9\%), es así que, del total de 233 menores, 104 son mujeres, aun cuando 4 instituciones se dedican al cuidado exclusivo de varones y únicamente 2 al cuidado de niñas. La mayor concentración de menores se encuentra en primaria (83.5\%) con una media de edad de 10 años y una desviación estándar de 2.8 .

La tabla 1 muestra las alfas de las escalas y las medias y desviaciones estándar de cada uno de los reactivos de la escala ELES. 
Tabla 1. Análisis de confiabilidad y frecuencia de ELES aplicada a los Educadores

\begin{tabular}{|c|c|c|c|c|}
\hline Variable & $\mathrm{N}$ & Media & D.E. & Alfa \\
\hline Actitudes & & & & .39 \\
\hline Mi trato cordial con los niños y niñas estimula su desarrollo. & 116 & 3.83 & .40 & \\
\hline Los niños y niñas del albergue no tienen capacidad para aprender. & 117 & 3.67 & .79 & \\
\hline Los niños no pueden enfrentarse a los desafíos que les plantea el sistema social. & 115 & 2.63 & 1.49 & \\
\hline La integración social favorece a los niños y niñas del albergue. & 115 & 3.70 & .80 & \\
\hline Funciones Educativas & & & & .62 \\
\hline Educativa & 117 & 3.50 & 1.03 & \\
\hline Informativa, de asesoramiento, orientadora. & 115 & 3.64 & .77 & \\
\hline De animación y dinamización de grupos. & 115 & 3.74 & .72 & \\
\hline Funciones Administrativas & & & & .86 \\
\hline Organización, planificación, programación, desarrollo y evaluación. & 113 & 1.22 & 1.64 & \\
\hline Gestión y administración. & 115 & .37 & 1.00 & \\
\hline Relación con otras instituciones. & 113 & .71 & 1.35 & \\
\hline Elaboración de programas. & 115 & 1.06 & 1.59 & \\
\hline Compromiso con la Propia Identidad & & & & .46 \\
\hline Siento que mi trabajo es bien recibido por mis compañeros y compañeras. & 117 & 3.49 & .83 & \\
\hline Les platico a mis familiares y amigos cuánto me gusta mi trabajo. & 115 & 3.65 & .81 & \\
\hline Siento que el pertenecer a esta área de trabajo me da algo más que dinero. & 116 & 3.90 & .30 & \\
\hline $\begin{array}{l}\text { Considero que mis compañeros/as y yo, nos sentimos identificados/as con nuestra } \\
\text { área de trabajo. }\end{array}$ & 117 & 3.68 & .67 & \\
\hline Habilidades Interpersonales & & & & 67 \\
\hline No entiendo realmente lo que los otros sienten. & 116 & 3.50 & .91 & \\
\hline Tengo problemas para defenderme a mí mismo/a. & 116 & 3.28 & 1.33 & \\
\hline Se me dificulta encontrar las palabras correctas para expresarme. & 117 & 2.98 & 1.42 & \\
\hline Me expreso bien verbalmente. & 117 & 3.56 & .83 & \\
\hline Percepción de Modificar el Desarrollo del Niño & & & & .65 \\
\hline Prevenir y compensar las dificultades de adaptación social de los niños y niñas. & 117 & 3.59 & .84 & \\
\hline Favorecer la autonomía del niño y niña. & 117 & 3.55 & .86 & \\
\hline Favorecer la participación de los niños y niñas. & 117 & 3.71 & .72 & \\
\hline Favorecer el desarrollo sociocultural, sociolaboral, institucional y comunitario. & 116 & 3.48 & 1.00 & \\
\hline Percepción de Modificar las Competencias del Niño & & & & .63 \\
\hline $\begin{array}{l}\text { Ayudar a potenciar la búsqueda de la información y comprensión del entorno social } \\
\text { de los niños y niñas. }\end{array}$ & 117 & 3.47 & .97 & \\
\hline
\end{tabular}

[Martha Esther SERRANO ARIAS \& Martha FRIAS ARMENTA] 


\begin{tabular}{|c|c|c|c|c|}
\hline Variable & $\mathrm{N}$ & Media & D.E. & Alfa \\
\hline $\begin{array}{l}\text { Desarrollar el espíritu crítico y la capacidad de comprensión y análisis de la realidad } \\
\text { de los niños y niñas. }\end{array}$ & 117 & 3.49 & .93 & \\
\hline Mejorar las competencias y aptitudes de los niños y niñas. & 117 & 3.65 & .75 & \\
\hline Lenguaje y Razonamiento & & & & .91 \\
\hline La estimulación a los niños para comunicarse es: & 116 & 4.47 & 1.34 & \\
\hline La utilización del lenguaje para desarrollar destrezas de pensamiento es: & 114 & 4.14 & 1.61 & \\
\hline Satisfacción Personal & & & & .84 \\
\hline La disposición para atender necesidades individuales del personal es: & 116 & 3.99 & 1.71 & \\
\hline El apoyo para necesidades profesionales del personal es: & 115 & 3.68 & 1.89 & \\
\hline Las oportunidades de crecimiento y carrera profesional dentro de la Institución son: & 115 & 3.43 & 1.92 & \\
\hline
\end{tabular}

Los resultados de la aplicación de la Evaluación de la Labor del Educador Social mostraron una confiabilidad de .80 (tabla 1).Aun cuando el alfa de dos dimensiones reportadas en la aplicación del ELES fueron menores a .60, se decidió conservarlas.
Las alfas de la escala de Conners se muestran en la tabla 2. Como puede observarse todas son mayores a .60 .

\begin{tabular}{|c|c|c|c|c|}
\hline Variable & $\mathrm{N}$ & Media & D.E. & Alfa \\
\hline Agresividad & & & & .91 \\
\hline Se comporta con arrogancia, es irrespetuoso & 232 &, 92 & .99 & \\
\hline Tiene explosiones impredecibles de mal genio. & 232 & 1.03 & 1.05 & \\
\hline Tiene aspecto enfadado, huraño. & 232 & .78 & .86 & \\
\hline Discute y pelea por cualquier cosa. & 232 & 1.08 & 1.07 & \\
\hline Acepta mal las indicaciones del profesor. & 232 & .90 & 1.01 & \\
\hline Conducta Oposicionista & & & & .74 \\
\hline Es impulsivo e irritable. & 231 &, 98 & 1.03 & \\
\hline Niega sus errores o culpa a los demás. & 231 & 1.12 & 1.06 & \\
\hline Problemas de Aprendizaje & & & & .80 \\
\hline Sus esfuerzos se frustran fácilmente, es inconstante. & 233 & 1.00 & 1.03 & \\
\hline Tiene dificultades de aprendizaje escolar. & 233 & 1.16 & 1.11 & \\
\hline
\end{tabular}

La figura 1 muestra los resultados del modelo. Se observa que el factor de Ambiente Institucional, formado por las variables de lenguaje y razonamiento, con peso factorial de .56 y satisfacción personal $(\mathrm{PF}=.58)$ tuvo una influencia directa y negativa sobre los problemas conductuales del 
niño, observado con un coeficiente estructural de -.34. Las competencias del Educador se conformaron sólo por seis de las siete dimensiones evaluadas: a) funciones educativas (peso factorial $=.75) ; \mathrm{b}$ ) habilidades (PF = .47); c) actitudes (PF = .38); d) compromiso con la propia identidad ( $P F=$ .51); e) percepción de modificar el desarrollo del niño ( $P F=.89$ ), y f) percepción de modificar las competencias del niño (PF $=.95)$ y tuvo una influencia directa y positiva (coeficiente estructural $=.30$ ) sobre Ambiente Institucional. Sin embargo, el efecto es indirecto y negativo sobre los Problemas Conductuales, a través del factor Ambiente Institucional puesto que la relación directa no fue significativa.

Los indicadores de bondad de ajuste fueron adecuados, proporcionados por el CFI y BBN$\mathrm{NFI}$ con valores arriba de .90, el BBNFI de .89 y el RMSEA con un valor de .07. El valor de la Chi cuadrada fue de 122 basado en 61 grados de libertad y con una probabilidad asociada de .oo. La $\mathrm{R}^{2}$ del modelo fue de .11, lo que significa que el modelo predice $11 \%$ de la varianza de la variable dependiente, esto es, de las Competencias del Educador.

\section{Discusión y conclusiones}

El objetivo fue evaluar los efectos de la formación de los educadores de los albergues infantiles con el ambiente y el comportamiento de los menores. Contrario a lo que se esperaba, la formación (que se operacionalizó como factor de Competencias del Educador), no parece afectar al comportamiento del menor de manera directa. Sin embargo, y de acuerdo también con la teoría, lo afecta de manera indirecta a través del ambiente que desarrolla dentro de los albergues, la formación de los educadores afecta directamente al ambiente y está a su vez la conducta de los menores. Esta relación entre ambiente y comportamiento se puede explicar desde la aportación de Cuevas del Real (2003) al mencionar al ambiente como un factor contextual asociado a la aparición de la conducta antisocial, en el cual el contexto, formado por el personal y el entorno es propuesto como factor de riesgo. También hay que recordar que la ambigüedad del rol del educador influye en el estrés profesional. La imprecisión de las tareas, debida a la falta de claridad acerca de los límites de su trabajo y de los recursos de la institución puede influir en el ambiente, positiva o negativamente, a través de la satisfacción de las necesidades del personal (Fernández-Millán, Hamido \& Fernández-Nava, 2008).

Con respecto a la primera hipótesis, en la que se esperaba que la formación del educador tuviera un efecto directo y positivo sobre el ambiente institucional, fue comprobada con los resultados del estudio. Sin embargo, con respecto a la segunda hipótesis en la que se esperaba un efecto directo y negativo de la formación adecuada del educador sobre los problemas conductuales del menor, no se encontró esa relación directa, pero si una indirecta a través del ambiente institucional.

Los hallazgos encontrados sobre la formación de los educadores concuerdan con Núñez (1993) en que las administraciones de los albergues contratan personas con todo tipo de perfiles profesionales, e incluso sin ellos. No obstante, $36.6 \%$ de los educadores tienen más de 5 años de antigüedad. Esto también es, en cierta manera, formativo. Basta recordar que toda educación social tuvo sus inicios en la práctica, más que en la reflexión teórica (Sáez, 2007) y que sus primeros educadores fueron voluntarios o profesionales en necesidad de capacitación para dicha tarea (Fermoso, 1999).

Las funciones fueron consideradas como una de las habilidades más importantes del Educador Social pues la delimitación que de éstas se haga define el perfil profesional y el resto de las competencias que requieran (Vallés, 2009). A pesar de que varios autores enumeran distintas y múltiples funciones para los Educadores de acuerdo a su área y población de intervención, se eligieron aquellas consideradas generales para la función desempeñada en los ámbitos aquí estudiados. Los educadores, sin embargo, indicaron que sus funciones están más relacionadas a funciones administrativas. Lo anterior puede deberse a que en los albergues con mayor población de educadores hay otro tipo de personal (pedagogos, psicólogos, o el mismo director de la Institución) encargado de la gestión, la relación con otras instituciones y la elaboración de los programas. Mientras que en albergues pequeños los mismos educadores deben encargarse de todas las áreas restantes de la casa (desde la regulación académica hasta el aseo y la cocina). Si bien la mayoría de los educadores están totalmente de acuerdo en que sus funciones son educativas, al contestar las preguntas varios de ellos comentaron no sentirse tanto como educadores, sino más bien como cuidadores. Tienen la concepción de que su labor es asegurar que el menor tenga cubiertas sus necesidades básicas pero no el formarlo o educarlo constantemente, tarea que atribuyen a otros profesionales relacionados con ellos, lo que se refleja en el ambiente del albergue. Esto es contrario a lo que algunos autores afirman (Cruz, 2009; Fernández del Valle \& Fuertes, 2007) pues en el caso de los albergues hermosillenses, el modelo de intervención no parece estar cambiando de uno asistencial a otro educativo. 
El compromiso con la propia identidad es una de las competencias transversales propuestas por ANECA (2005). Esta capacidad ayuda al educador a "reconocerse y valorarse como profesional que ejerce un servicio a la comunidad" (p. 146) y que se preocupa por su actualización. Los educadores, al contestar las preguntas sobre su identidad aseguran sentirse identificados con su trabajo, realizarlo por incentivo más altruista que monetario y sentir que son apreciados por sus compañeros. Sin embargo, en conversaciones previas y posteriores a la aplicación de la Escala, más de la mitad coincidían en sentirse poco considerados por sus superiores en cuanto al manejo y toma de decisiones sobre la vida de los menores y poco valorados, ya que sienten que no son suficientemente remunerados tanto económica como emocionalmente por la institución. Esto se muestra en oposición a lo presentado por la Escala, pero más en relación a lo descubierto por Cruz (2009) en cuyo estudio las educadoras mostraban sentimientos de infravaloración, desamparo y desprotección, mucho de lo cual tenía su origen en la falta de una formación específica. También presentaron, en coincidencia con Ayerbe (2000), inmovilismo, ausencia de perspectivas de promoción y una falta de confianza en sí mismos, todos los cuales se mencionan como resultado de una falta de identidad profesional y que pueden repercutir en la calidad del ambiente.

Entre las respuestas a las que habría de prestarse mayor atención, se encuentran las referentes a las actitudes puesto que la mayoría no creen que los menores del Albergue tengan capacidad para aprender o que puedan enfrentarse a los desafíos que les plantea el sistema social. Esto puede resultar dañino para el desempeño de su labor ya que una de las características básicas de este tipo de personal ha de ser la aceptación de las personas y la creencia de la necesidad de cambio social (Touza \& Segura, 2001). Sin mencionar que el trabajo educativo está vinculado a la ideología con la que el educador interpreta su realidad (Cruz, 2009) y que el tipo de relación que desarrolle con el menor dependerá de la imagen que tengan de él (Melendro, 2007). Resulta importante hacer notar que entre el $40 \%$ y $44 \%$ de quienes expresaban la creencia en la falta de capacidad de los menores fueron aquellos que tenían licenciaturas o carreras técnicas relacionadas con la educación. Quienes menos lo juzgaban así, eran los educadores que sólo habían estudiado hasta la primaria. Esto concuerda con que no sólo la formación profesional garantiza la adecuada labor socioeducativa (Santibañez, 1999). Cabe mencionar que la mayoría de los educadores con menor formación profesional tienen más antigüedad.
Aquellos un poco más profesionalizados fueron contratados más recientemente.

Se pueden apreciar de aquí dos cosas. Primero, que con el transcurso del tiempo se fue tendiendo hacia la contratación de un educador más profesionalizado de acuerdo a las recomendaciones internacionales (Gobierno de Brasil, 2007; UNICEF, 2009a) y nacionales después (Cámara de Diputados, 2009; Castro, 1996; CEDH, 2006). Segundo, se podría pensar que, puesto que los educadores con menor formación profesional tienen mayor antigüedad, han tenido también más experiencias de historias de éxito entre los menores que han atendido, lo que les permitiría tener una actitud más positiva hacia ellos. Sin embargo, esta hipótesis queda abierta para futuras investigaciones.

Las habilidades interpersonales también son una competencia transversal definida a grandes rasgos como "la capacidad de relacionarse positivamente con otras personas"(ANECA, 2005, p. 145) a través de la empatía y la expresión asertiva, importantes ambas para facilitar la relación de confianza y creación de vínculos afectivos con los menores (García-Barriocanal, et al., 2007). Como se pudo observar en la tabla 1, la mayoría de los Educadores tienen problemas para demostrar empatía y de éstos, arriba del $40 \%$ fueron los educadores con licenciaturas relacionadas con esta labor. Esto presenta un problema pues la comunicación inadecuada es una de las características de lo que Cuevas del Real (2003) define como violencia institucional. Es también uno de los elementos que el educador social debe poseer en vistas a facilitar una adecuada interacción con los menores (Redondo et al., 1998) y su adecuado proceso de ajuste emocional (Gobierno de Brasil, 2007; Vallés, 2009).

Un alto porcentaje de educadores mencionaron no entender lo que otras personas siente. Esto resulta un descubrimiento importante puesto que con la sensibilidad que el educador posea para notar las necesidades del menor, se pueden percibir y paliar los comportamientos problemáticos e incluso antisociales de los menores (Cuevas del Real, 2003). Además, una implicación emocional ayuda a establecer vínculos que dejarán huella (AIEJI, 2005). Sin mencionar que la carencia de habilidades sociales en los educadores, así como el manejo de la propia vida emocional interfiere con la habilidad del niño y adolescente para crear relaciones adecuadas en el futuro (Booth, Spieker, Bernard \& Morisset, 1992).

La mayoría de los educadores consideran que el albergue sí estimula a los menores a comunicarse y utiliza el lenguaje para desarrollar destrezas de pensamiento en ellos. Esto refuerza lo 
mencionado por Mondragón y Trigueros (2004) en cuanto a que el potencial evolutivo de un entorno aumenta cuando se permite y estimula la participación, tanto con los educadores como entre los mismos menores. Un ambiente sano, puede ser un factor reductor de la aparición de trastornos en los menores (March, 2007).

El indicador de satisfacción del personal se pensó importante puesto que algunos especialistas consideran que las condiciones laborales inadecuadas pueden tener una influencia negativa en el desarrollo de la práctica educativa (Cruz, 2009). Esta situación puede tornar el ambiente en poco acogedor y a los menores vulnerables (Melendro, 2007). Además de que los educadores requieren sentirse respetados en sus centros de trabajo y que tanto sus necesidades, como sus dificultades y logros sean reconocidos (Barudy \& Dantagnan, 2005); lo que finalmente evitará el desgaste profesional y los efectos indirectos que éste pudiera tener en los menores. La mayoría de los educadores describieron de Buena a Excelente la disposición de la institución para atender sus necesidades individuales.

El que los educadores consideren el ambiente institucional como más que bueno pudiera explicar por qué se descubrieron relativamente pocos problemas conductuales en los menores. No se puede decir que estos datos concuerden con los hallazgos de otros estudios pues no se tiene referencia del comportamiento de los menores albergados en años anteriores. Tampoco se analizaron aquí problemas emocionales o conductas delictivas. Sí se puede, sin embargo, tomar estos datos como referencia para futuras investigaciones.

Es necesario recordar que esta población, por sus mismas características, llega a la institución con menor adaptación académica, deficiencias en sus habilidades sociales, desorden por estrés postraumático, daño en su autoestima, agresividad, entre otros (Gómez, 1996; Gaxiola \& Frías, 2008). La formación de los educadores, y principalmente su especialización, se hace entonces más urgente para que puedan, no sólo contener dichas manifestaciones, sino prevenirlas, así como corregirlas.

La profesión del educador social refleja su propia historia (Vallés, 2009). Lo hizo en Alemania, en España y lo hace en América Latina. Los resultados de este estudio son prueba de ello puesto que la especialización del educador en esta rama de la educación no sale de las universidades sino de la propia práctica de cientos de personas dispuestas a aprender a educar y cuidar a los niños y adolescentes institucionalizados. Igualmente, nos indican que los albergues manejan un personal escaso, de edad avanzada, y con pocos estudios. Además, a raíz de este estudio se encontró que la población de educadores es poco equitativa en cuanto a género ya que hay más mujeres que hombres.

Puesto que la Pedagogía Social es considerada como reflexión y disciplina científica (Cruz, 2009), es la opinión de los investigadores que esta ciencia, como lo ha hecho al introducirse en países sud americanos, se verá enriquecida al operar como aparato teórico sobre el cual se fundamente la labor de profesionales que tienen años de experiencia pero que hasta este momento no han sido reconocidos. Una vez los educadores mexicanos tengan las bases educativas necesarias para continuar con su trabajo dentro de los albergues infantiles, podrán ser reconocidos como tales y no meros cuidadores. A partir de aquí podrán aportar a la Pedagogía Social con la riqueza de los conocimientos aprendidos en años de experiencia y analizados ahora bajo la luz de un marco teórico apropiado.

A pesar de que este estudio no ahondó en todas las competencias sugeridas por ANECA, sí se sugiere su estudio y profundización como contenido de un curso de formación para los educadores. Se sustenta la idea de que éstos educadores podrían contar con más herramientas para el desarrollo eficaz de su labor al ser formados en una teoría educativa pertinente (AIEJI, 2005; ANECA, 2005; Sáez, 2005), posiblemente a través de la creación de una licenciatura en Educación Social o grado académico de posgrado.

En este estudio se les englobó a todos los tipos de albergues en una sola categoría; se reconoce que estas variables pueden haber influido en los resultados. Tampoco se pudo tomar en cuenta el tiempo de estancia de los menores ya que algunos albergues registran los reingresos como nuevos ingresos, lo que no permite tener los datos estadísticos reales. A pesar de todo esto, se considera que sí se alcanzaron los objetivos con los que inicia esta investigación. Se identificaron las características de formación de los educadores, así como los comportamientos de los menores y de qué manera influyen la formación y el ambiente en dichos comportamientos. En aras de mejorar la labor educativa de los albergues, los resultados de este estudio permiten establecer la necesidad de continuar con investigaciones que busquen dar luz a todas las interrogantes aun pendientes. 


\section{Referencias bibliográficas}

Agencia Nacional de Evaluación de la Calidad y Acreditación (ANECA). (2005). Libro Blanco. Título de grado en pedagogía y educación social. Volumen 1. España: ANECA.

Arruabarrena, M. (2001). Evaluación y tratamiento familiar. En J. De Paul \& M. Arruabarrena (Eds.). Manual de protección infantil (297-340). Barcelona: Massons.

Asociación Internacional de Educadores Sociales. (AIEJI). (2005). Plataforma para las educadoras y educadores sociales en Europa. España: AIEJI. Recuperado de http://www.ceesc.cat/index2.php?option=com_docman\&task=doc_ view\&gid $=75 \&$ Itemid $=403$

Asociación Internacional de Educadores Sociales. (AIEJI). (2007). Estatutos de AIEJI. Barcelona: AIEJI. Recuperado de http://www.aieji.net/attached/bylaws/By-Laws2007SFinal.pdf

Ayerbe, P. (2000). La figura del educador social y otros profesionales de la educación. En P. Amorós \& P. Ayerbe (Eds.), Intervención educativa en inadaptación social, (55-81). Madrid: Síntesis.

Barudy, J. (2005a). La ecología social de los buenos tratos infantiles. En J. Barudy \& M. Dantagan (Eds.), Los buenos tratos a la infancia (43-52). Barcelona: Gedisa.

Barudy, J. (2005b). Las necesidades infantiles. En J. Barudy \& M. Dantagan (Eds.), Los buenos tratos a la infancia (61-75). Barcelona: Gedisa.

Barudy, J. \& Dantagnan, M (Eds.). (2005). Un modelo terapéutico basado en el buen trato y el apoyo a la resilencia. En Los buenos tratos a la infancia (213-244). Barcelona: Gedisa.

Booth, C., Spieker, S., Bernard, K. \& Morisset, C. (1992). Infants at Risk: the Rule of Preventive Intervention in Deflecting a Maladaptative Development Trayectory. En J. McCord \& R. Trambley (Eds.), Preventing Antisocial Behavior (2142). Nueva York, E.U.: Gulford Press.

Bravo, A. \& Fernández del Valle, J. (2009). Crisis y revisión del acogimiento residencial. Su papel en la protección infantil. Papeles del Psicólogo, 30(1), 42-52.

Cámara de Diputados. LX Legislatura. (2009). Iniciativa de Diputados del PRI para operación de Casas Hogar. Carpeta Informativa. Coordinación General de Comunicación Social. Pp. 15-16. Recuperado de www.cddhcu.gob.mx.

Camors, J. (2005). Educación social: una perspectiva desde y para América Latina. Conferencia Inaugural del XVI Congreso Mundial de Educación Social. Montevideo, Uruguay. Recuperado de http://www.projoven.gub.uy/Documentos/Congreso\%2Oeducadores/1_Jorge_Camors.doc.

Castro, P. K. (1996). Un Problema que a Todos Incumbe. En Congreso Nacional sobre Maltrato al Menor. Memorias. 26, 27 y 28 de noviembre 1995 (26-28). México: Sistema Nacional para el Desarrollo Integral de la Familia.

Doménech, V., Esbrí, J.V., González, H.A. \& Miret, L. (2004). Actitudes del profesorado hacia el alumnado con NEEDD. IX Jornadas de Fomento de la Investigación en Ciencias Humanas y Sociales. Universitat Jaume I. Castellón.

CEDHJ. (2006). Evidencia CEDHJ deficiencias en albergues infantiles. DHUMANS, 11(10), 1-4. Guadalajara, México.

Conners, K. (2008). Conners Manual. Toronto, Canada: Multi-Health Systems.

Cruz, L. (2009). Infancias y educación social: prácticas socioeducativas en contextos residenciales de protección en Galicia (Tesis doctoral no publicada, Universidad de Santiago de Compostela, Santiago de Compostela, España).

Cuevas del Real, M. (2003). Los factores de riesgo de la prevención de la conducta antisocial. En A. Silva (Ed), Conducta Antisocial: un enfoque psicológico (25-64). México: Pax.

Fermoso, P. (1999). Seminario/Taller del practicum en la Diplomatura de Educación Social. En F. Esteban \& R. Calvo de León (Eds.), El practicum de la formación de Educación Social (85-106). Burgos: Servicio de Publicaciones de la Universidad de Burgos.

Fernández del Valle, J. \& Fuertes, J. (2007). El acogimiento residencial en la protección a la infancia. España: Pirámide.

Fernández-Millán, S., Hamido, A. \& Fernández-Nava, M. (2008). El educador social de menores. Cuaderno de aprendizaje. Madrid: Pirámide.

Fuertes, J. \& Fernández del Valle, J. (2001). Acogimiento residencial. En J. De Paul \& M. Arruabarrena (Eds.), Manual de protección infantil (409-470). Barcelona, España: Massons.

García-Barriocanal, C., De la Herrán, A. \& Imaña, A. (2007). El acogimiento residencial como medida de protección. Madrid, España: Defensor del menor en la Comunidad de Madrid.

Gaxiola, R. J. \& Frías, M. (2008). Un Modelo Ecológico de Factores Protectores del Abuso Infantil: un Estudio con Madres Mexicanas. Hermosillo, México: Resma.

Gobierno de Brasil (2007). Anteproyecto de Naciones Unidas para el uso apropiado y condiciones del cuidado alternativo de niños/as. Aprobada en la Asamblea General de Naciones Unidas el 20 de noviembre de 2009. Recuperado de http://www.crin.org/docs/DRAFT_UN_Guidelines.pdf.

Gómez, I. E. (1996). Secuelas Psicológicas del Maltrato y el Abuso Sexual. En Congreso Nacional sobre Maltrato al Menor. 26, 27 y 28 de noviembre 1995 (38-55). México: Sistema Nacional para el Desarrollo Integral de la Familia. 
González, M. (2010). Cárceles Infantiles. Proceso. Semanario de Información y Análisis, 1740. Suplemento Jalisco, 278, pp. I-VI.

Gribble, K. (2007). A model for caregiving of adopted children after institutionalization. Journal of Child and Adolescent Psychiatric Nursing, 20(1), 14-26. doi: 10.1111/j.1744-6171.2007.00076.x.

Harms, T., Clifford, R. \& Cryer, D. (2005). Early Childhood Environmental Rating Scale-Revised (ECERS-R). New York: Teachers College Press.

Hernández, J. (2014). Transcripción de Los niños de la calle. Recuperado de https://prezi.com/dgjxmzmtxgtt/los-ninosde-la-calle/.

INEGI. (2010a). Principales resultados del Censo de Población y Vivienda. Recuperado de http://www.inegi.gob.mx/ prod_serv/contenidos/espanol/bvinegi/productos/censos/poblacion/2010/princi_result/cpv2010_principales_resultadosl.pdf.

INEGI. (2010b). Módulo de Trabajo Infantil 2009. Encuesta Nacional de Ocupación y Empleo 2009. Documento Metodológico. Recuperado de http://www.inegi.org.mx/est/contenidos/espanol/metodologias/encuestas/hogares/ docto_met_mtiog.pdf.

INMUJERES. (2006). Encuesta de Maltrato Infantil en Adolescentes de Secundarias en cuatro estados de la República Mexicana. Recuperado de http://cedoc.inmujeres.gob.mx/documentos_download/100769.pdf.

La BUAP y la U. de G. signarán convenio en educación a distancia. (2005). UNIVERSIA. Recuperado de http://noticias. universia.net.mx/vida-universitaria/noticia/2005/03/22/106510/buap-u-g-signaran-convenio-educacion-distancia. html.

Ley de Asistencia Social del Estado de Sonora (1986). Gobierno del Estado de Sonora.

March, R. (2007). Claves para la intervención con menores acogidos en recursos residenciales que presentan conductas problemáticas. Intervención Psicosocial, 16(2), 213-227. http://dx.doi.org/10.4321/S1132-05592007000200006.

Melendro, M. (Dir.) (2007). Estrategias educativas con adolescentes y jóvenes en dificultad social. El tránsito a la vida adulta en una sociedad sostenible. Madrid, España: UNED.

Mondragón, J. \& Trigueros, I. (2004). Intervención con menores. Acción socioeducativa. Madrid, España: Narcea.

Nesmith, A. (2006). Predictors of running away from family foster care. Child Welfare, 85(3), 585-609. doi:10.1016/j. childyouth.2009.06.003

Núñez, V. (1993). El educador especializado. En J. Sáez (Coord.), El Educador Social (125-142). Murcia: Universidad.

Núñez, V. M. (2008). Intervención Interdisciplinaria para una Niñez Vulnerable y Cautiva, (Tesis de maestría no publicada, Universidad de Sonora, Hermosillo Sonora México).

Ortega, J. (2005). Pedagogía social y pedagogía escolar: la educación social en la escuela. Revista de Educación, 336, $111-117$.

Papalia, D., Wendkos, S. \& Duskin, R. (2001). Desarrollo Humano. Bogotá: McGraw-Hill Interamericana.

Petrus, R. (1993). Educación Social y perfil del educador social. En J. Sáez (Coord.), El Educador Social (165-214). Murcia: Universidad.

Petrus, R. (Coord.). (1997). Concepto e Educación Social. En Pedagogía Social (63-94). Barcelona, España: Ariel.

Redondo, E., Muñoz, R. \& Torres Gómez. (1998). Manual de buenas prácticas para la atención residencial a la infancia y adolescencia. Madrid, España: FAPMI.

Rubin, R. B. \& Martin, M. M. (1994). Development of a measure of interpersonal communication competence. Communication Research Reports, 11, 33-44.

Sáez, J. (2005). La profesionalización de los educadores sociales: construcción de un modelo teórico para su estudio. Revista de Educación, 336, 129-139.

Sáez, J. (2007). Pedagogía social y educación social. Historia, profesión y competencias. Madrid, España: Pearson Educación, S.A.

Santibañez, R. (1999). El seguimiento en el practicum de Educación Social. En F. Esteban \& R. Calvo de León (Eds.), El practicum de la formación de Educación Social (189-200). Burgos, España: Servicio de Publicaciones de la Universidad de Burgos.

Touza, C. \& Segura, M. (2001) Cuestionario de evaluación de la formación del educador social. Educació y Cultura: Revista mallorquina de pedagogía, 14, 67-85.

Unicef. (2009a). Progreso para la Infancia: Un balance sobre la protección de la niñez. UNICEF. Recuperado de http:// www.unicef.org/spanish/progressforchildren/files/Progress_for_Children-No.8_SP_081309.pdf.

Unicef. (2009b). Conmemoración de los 20 años de la Convención de los Derechos del Niño. UNICEF. Recuperado de http://www.unicef.org/spanish/publications/files/SOWC_Spec._Ed._CRC_Statistical_Tables_SP_111809.pdf.

Vallés, J. (2009). Manual del educador social. Madrid, España: Pirámide.

Vélez, C. (2006). La pedagogía Social en Colombia, (Tesis doctoral no publicada, Universidad de Educación a Distancia, Madrid, España). 


\section{CÓMO CITAR ESTE ARTÍCULO}

Frias, M. \& Serrano. M. (2017). La formación de los educadores en albergues infantiles en México. Factor de riesgo para la conducta antisocial de los niños. Pedagogía Social. Revista Interuniversitaria, 29, 197-210. DOI: 10.7179/PSRI_2017.29.14.

\section{DIRECCIÓN COMPLETA DE LOS AUTORES}

Martha Frias Armenta: Correo Electrónico / E-mail:marthafrias@sociales.uson.mx.

Martha Esther Serrano Arias: Sección Calabria, Villa Croata \#17, Villas del Mediterráneo, Hermosillo, Sonora, México, CP 83220 Correo Electrónico / E-mail: me-serrano@hotmail.com.

\section{PERFIL ACADÉMICO}

Martha Frias Armenta: Profesora titular del departamento de Derecho de la División de Ciencias Sociales de la Universidad de Sonora. Licenciada en derecho por parte de la Universidad de Sonora, obtuvo la maestría y el doctorado en la Universidad de Arizona en el área de Psicología Jurídica y es miembro del Sistema Nacional de Investigadores, nivel III y Perfil PROMEP. Además, forma parte del cuerpo académico consolidado problemas sociales. La línea de investigación conducta antisocial y delictiva, incluyendo violencia intrafamiliar, delincuencia juvenil, justicia restaurativa, justicia procedimental y distributiva y delito ecológico. Además, es evaluadora de proyectos de investigación en los Consejos de Ciencia y tecnología de México y España y funge como editora de varias revistas nacionales e internacionales. Ha sido nombrada como investigadora distinguida de la División de Ciencias Sociales de la Universidad de Sonora por varios años. Cuenta con varias publicaciones en revistas indexadas a nivel nacional e internacional y 6 libros. Ha sido conferencista invitada en varias Universidades Nacionales y extranjeras. Fue asesora externa del Instituto Sonorense de la Mujer del Estado de Sonora durante 2005 y directora general del Instituto de Tratamiento y Aplicación de Medidas para Adolescentes en conflicto con la ley del Estado de Sonora del 2009 al 2011.

Martha Esther Serrano Arias: Cursando Doctorado en Ciencias Sociales incorporado al PNPC de Conacyt con la Tesis "Estudio Transcultural de Inteligencia Social: el caso de México y Alemania", Universidad de Sonora - Hermosillo, Son. Diplomada en Gerontología DEG I: Nivel 1. Maestría en Innovación Educativa incorporado al PNPC de Conacyt y licenciada en Pedagogía. 
